\title{
The Development of Chinese Vedio Games Visual Before 21 Century
}

\author{
Xiao Yuan \\ Department of Art, Southwest University for Nationalities, Chengdu City, China \\ Email address: \\ ruluca@foxmail.com

\section{To cite this article:} \\ Xiao Yuan. The Development of Chinese Vedio Games Visual Before 21 Century. American Journal of Art and Design. \\ Vol. 3, No. 1, 2018, pp. 1-4. doi: 10.11648/j.ajad.20180301.11
}

Received: April 8, 2018; Accepted: April 24, 2018; Published: May 15, 2018

\begin{abstract}
In this paper, the Electronic Game or Video Game to the formal stage of development in the early years of the development course of audio-visual, cause and the main factors and the development and the social environment and requirements to do certain bolt. To the emergence of video games and nintendo games on TV and the development of the PSP, for example, will the initial game to promote children develop good habits, help the elderly brain flexible operation, the purpose of through interactive and comprehensive characteristics of the game itself, prompting the another reform of visual art. Combined with experience of Nintendo, the game development process and problems of China.
\end{abstract}

Keywords: Video Game, Visual Effects, Game Development

\section{The Emergence of Electronic Games and Visual}

Since the $1960 \mathrm{~s}$ by Cambridge university computer scientists A. S. Douglas in 1952 developed the first recorded in the history of computer games Noughts \& Crosses, this is a tic-tac-toe game can be run on a computer, then the world's first interactive game Tennis for Two ping-pong game with the American Hungarian mathematician John Kemeny developed a computer programming language [1], video game entertainment began as a form of entertainment in social life plays an indispensable part of.

When the first game listed the promotion of the journal Computer Space, this through some remote control equipment and Computer and TV do interactive entertainment is accepted by the people, the original video games have a common characteristic: separate, of point, line and plane can be the role of the handling of a certain point and line on the television screen as the lever commands and programs to do the corresponding interaction [2], the digital world of black and white dot, simple design can make the dimensional feeling of different levels and the cycle of repetitive levels increase the playability and overhand, background music of binary bit rate also can form good melody such Settings will video games the sense with the fresh will throughout the game lively and interesting.
With the continuous improvement of computer computing and programming technology, the Japanese game developer Namco developed in 1980 was named "the pac-man" (figure 2) host color game, this means that the video game screen and is more than just the color is the original design of "promoting the children develop good habits, to help the old man flexible of the brain in action", the purpose of completely when the commercial category after intervention and the interests of the game to create value $[2,3]$, a lot of competition to make electronic game industry by leaps and bounds, different companies have different technology, electronic game audio-visual experience became absolute competitive game makers around the world and the development direction.

Electronic games variability is different from the conventional art of film and television art and music art form, is limited to the audio-visual language under the conventional art form is mainly composed of the artistic creation of art creators to convey to the audience the theme of the core content, stay on the experience in visual images and sounds, in diversified functional function of video games, not only contains the seeing and hearing elements, Is the biggest advantage of Interactivity, Interactivity, interaction and game software script writing good plot, via voice, external equipment and related operation mode to the plot of the Play experience video Games, has more than the end of the plot and different experience, is the basic standard of video Games as a contemporary times times, from the direction of cultural 
development trend analysis, video Games and film art fusion can to represent the variable characteristics, such as Guerrilla Games released in February 2017 in Sony Play Station 4 platform open world Games the Horizon: Dawn (Horizon: Zero Dawn), this game fusion the future science fiction elements, from the picture directly equivalent to the effect of CG animation film, similar to the concept of electronic game types derived from time time to try, such as "call of duty modern warfare trilogy, crysis far cry" Dawn, fade "GTA5 (Grand Theft Auto V) etc, by the frost, fantasy, and the Unity of game development engine development and the development of the hardware facilities, electronic Games the variability of better fusion of modern and traditional art form of cash.

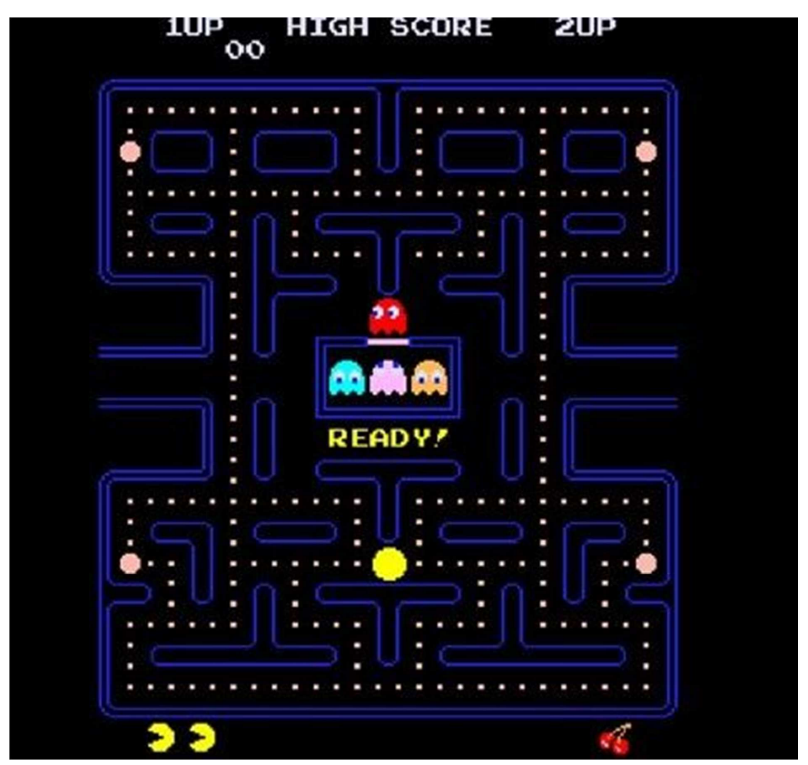

Figure 1. The pac-man.

\section{Nintendo with the Advent of the Game}

In the $1980 \mathrm{~s}$ Japanese electronics maker Nintendo
(Nintendo) starts to make the world famous "big king kong", "tetris" and so on with red and white machine (Family Computer) after the home consoles, there are a large number of excellent art designers will Nintendo video game picture did it at the time the best embodiment of science and technology. Relying on the eight digital chip technology innovation, nintendo launched in 1983, the famous work "super Mario", the game screen and music until today still is irreplaceable masterpieces: color TV rich color performance, the graphics of watercolor painting, corresponding to the buildings, plants do setting is unique, the role of art and vivid, the action of cohesion and control are on the creation of the future for nintendo buried under a heavy sum.

Nintendo created the world's first LCD screen handheld game (Game \& Watch) in 1980. The introduction of the LCD panel, exquisite pictures and fluency, for game art design of the cohesion of the suit makes the video games becomes vivid picture. In 1989 by nintendo's designers horizontal Wells army created a handheld historical milestone activities: ping introduced to surprise the world Game Boy [4, 6](hereinafter referred to as the "GB PSP), the electronic gaming platform spread to carry can also enjoy the Game, at the same time, the images of delicate began to the position of nintendo in the Game world.

Count of nintendo's classic book, there is no lack of found that nintendo game visual exploration process and the development of, for example, in 1992 the login GB platform Kirby (figure2) series, from now's point of view, than the first star card is so rough, simple circle of roles in a fantasy adventure story, feeling seems to be low age groups, but the result on the contrary, idiot is not equal to is simple, easy leisure metamorphosis, brings the very cordial atmosphere of fun, lovely modelling simple adds new Mode on the basis of Extra Mode, this Mode allows the playability of the game is considerable increase, at the same time the work will also be nintendo's main screen style and game pad under the direction of his works in the future.

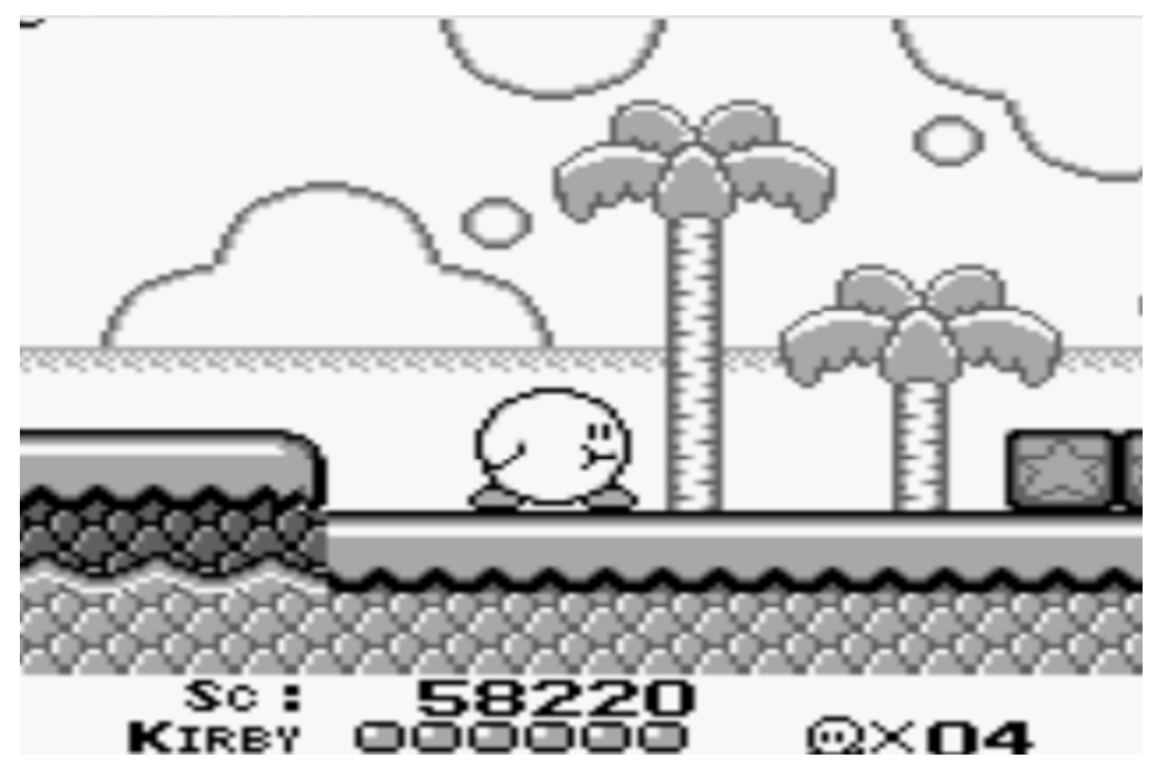

Figure 2. Kirby 
Japanese game makers Nintendo (Nintendo) were published on January 17, 2018 is applied to video game released in March 2017 host Nintendo Switch of a new model of combining Virtual Reality and practical (Do It) of the game application (as shown in figure 3,4), the game fully defined electronic games "variability": by joining together of different style of paper mould, cooperate hand made of different modules and unit, then to exclusive to the Switch can be spliced type operating system, infrared sensory system with camera, for specific game experience can be achieved practical content; Nintendo games "targeted" from FC host Numbers game "super Mario" (SuperMario) is based on the development of the whole age, and later developed by most of the targeted mainly at low age games (EC: Early Childhood or E: Everyone).

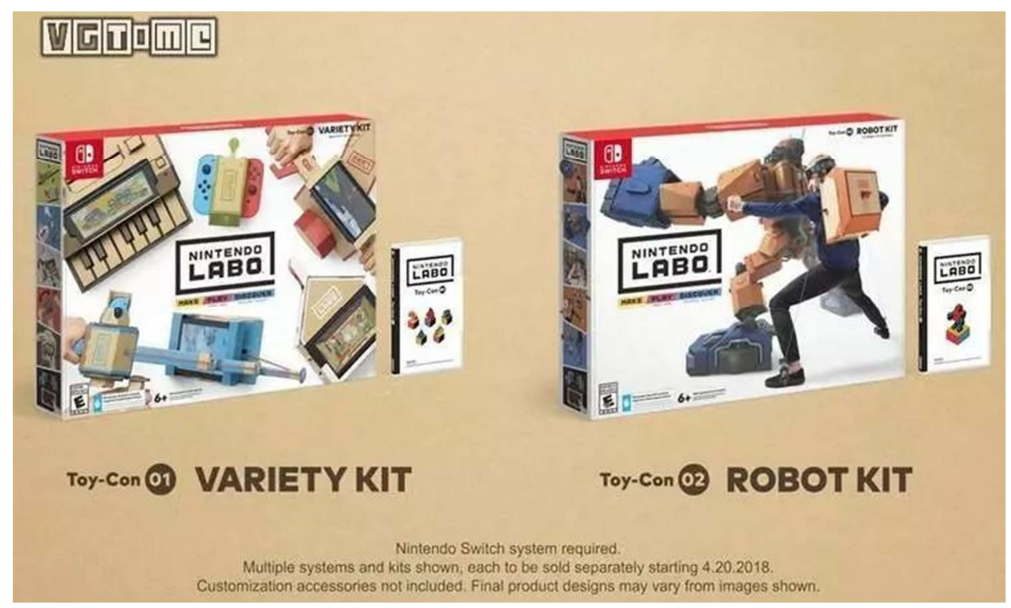

Figure 3. The Game: Do It.

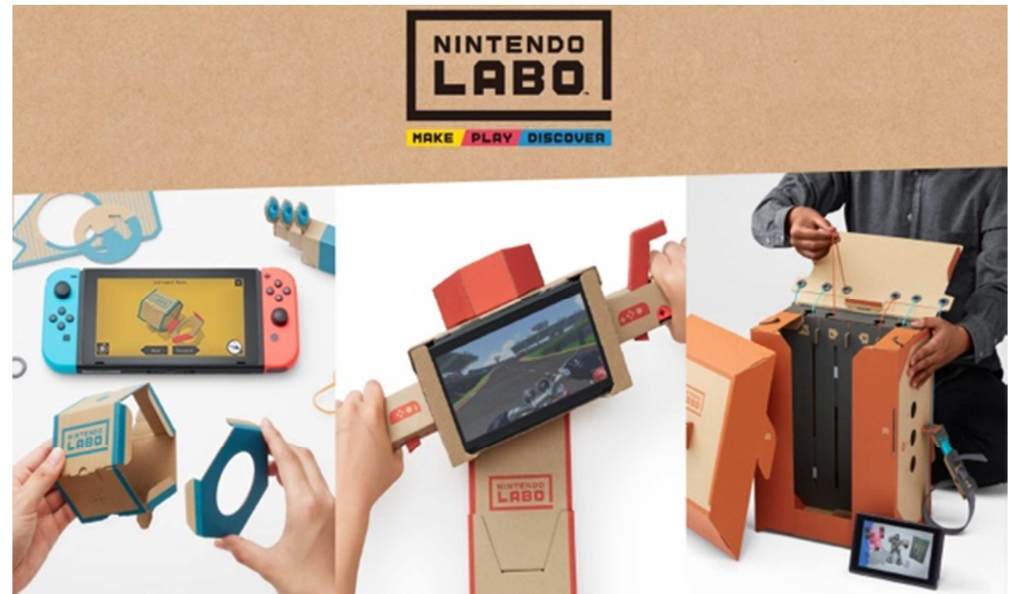

Figure 4. The Game: $L A B O$.

When handheld video games became popular, more and more Game makers began a new round of market competition, [5] a large number of excellent works of literature and art competition, nintendo company in 1996 to launch a role-playing Game as well as "fairy treasure can dream" (Pokemon), the work development up to now, the official has 53 Game, inherited the nintendo fun style, the role of the Game to join the drama is no inferior with SQUARE: company (SQUARE ENIX CO., LTD) final fantasy series, Pokemon design prototype to catch insects and popular Japanese teenagers practice Game, a lot of visuals of the Game and scene, assemble graphic LCD pixels on the platform into attractive images, two-dimensional and three-dimensional combination of the role of the rich and fascinating story, prompting the Game world sale after Game "super Mario", as the end of the nineteenth century a new generation of nintendo host GBC (Game Boy Color), Color LCD screen on a peak again in electronic gaming platform, different from the black and white LCD part adopts the thin film transistor TFT LCD, can show 58 in 32000 Color Color, more mainly TFT display image no shadow, this is before, according to GB, therefore, for some of the pass and fighting Game can also be clearly displayed. Different game screen can show the amazing effect, its quality as MD (sega's color PSP) [7].

Which have become heaven game company in the creation of independent game at the same time, will not only features excellent image and the original content, continuous improvement and development at the same time also learned a lot of material in the field of life and art effect, in the process of experience of the game, through visual images and music 
interact to get the story of process flow, will the original film can achieve the unique experience combined with the freedom interaction, form the unique charm of video games.

\section{Development and Problems of Chinese Electronic Games}

The emergence of electronic games has enriched the pastime, idle time in the current conditions of the rapid development of economy in China, video games also occupy a considerable market share. Chinese game originally formed in Taiwan province, in the mid - ninety - s has formed a relatively complete industrial chain, when the mainland market development, due to the lack of early Chinese domestic intellectual property rights concept, resulting in a large number of pirated games and copied content of the story, because the real necessities of original content, can lead to the development of domestic electronic games market stagnate, between 1994 to 1996 [10, 5, 8], created by the gold plate company seals commandos and two years later, the determination of the prefect of opening up China's video game industry circle, after the formation of Chinese electronic games official guidance on track.

Is the highest peak of the Chinese game industry from 1997 to 1997 , by China Taiwan daewoo co., LTD. During the period of Yao Zhuangxian, Xie Chonghui and others formed fanatics studio offering Chinese single machine game is pinnacle "xianjian wonder biography", the creation by the theme of Chinese traditional martial arts style computer LAN games, successfully learned Japanese role-playing game (Role playing), $[3,7]$ the advantages of blend in the plot of the story, $[9,6]$ in the ninety s has been strengthened by the drawback of the lack of domestic game in scope of resistance to play. But in essence, this is also so far unable to appear on the market in China and international large standalone game manufacturers can produce one of the reasons for competitiveness, rough, the role of originality and domestic large-scale tradition of "plagiarism", leading to the domestic game industry can only stop at the exploratory stage. And the emergence of online games has greatly strengthened the domestic game market, from the initial "Stone Age" to "the legend" so far the domestic network game empire, tencent, netease, etc in the Chinese market to occupy and lay the absolute position of online game players in China, Chinese domestic stand-alone game began to crumble.

Why domestic stand-alone game always couldn't do the competitive work? First, visual effects, as a game, excellent visual experience is absolutely the core game, game by game programming and production of the original comes mainly from the artists, but due to domestic vicious competition, a large number of plagiarism and piracy to profit for the purpose of game bad businessmen lose the original market competitiveness, so that the game art development so far only technology and lack of creativity. Secondly, the plot, as the pillar of the game soul exists, a game can attract consumers mainly by is a story of the script $[5,8]$, as a strong heritage of
Chinese traditional culture, had a lot of factors can be created with the traditional story, but deliberately to imitate foreign companies work and give up the advantages of itself, is not desirable. Thirdly, time costs due to the requirement of rapid economic value, the best work is often just a flash in the pan is spent in the game market, due to time is proportional to the cost and create value relations, under the so-called system requires the appropriate time to make good films is very difficult.

\section{Conclusion}

China electronic game not just limited to obtain economic effect, contrast can be found throughout the history of electronic games early, the role of the game is not only a entertainment properties, can be spread by means of game culture or tradition, or language learning new knowledge, aesthetic progress through the game, Chinese game plays find that including Japan nintendo, SONY, American blizzard, sega game giant such as at the beginning of the original problem and the problems during the development of Chinese game is the same, which can be concluded that with the development of the Chinese game process, the improvement of original although it is difficult to conquer the problem, but as the technology upgrade and the emergence of outstanding talent, as Japanese game innovations of the last century, it will step to the right track and create brilliant.

\section{References}

[1] Sina news. Nintendo FC 30th anniversary, 2013: p46-49.

[2] BuWei. Journalism and communication research, 1996: p14-18.

[3] Ray-michel. Jian. Movies are not pure sex - films and video games, the world film, 2005: p19-20.

[4] Wang Chen. China needs great wisdom game, software Tribune, 2004: p12-18.

[5] Talib, Zawawi A. Design and development of an interactive virtual shadow puppet play [C]. Malaysia: Lecture Notes of the Institute for Computer Sciences, Social-Informatics and Telecommunications Engineering, 2011: p118-126.

[6] Ning, Shurong. Using vector mapping and inverse kinematics for motion retargeting [C]. Beijing: Communications in Computer and Information Science, 2012: p748-756.

[7] Jing, Chenglin. Fuzzy adaptive algorithm for biped robot inverse kinematics [C]. Chongqing: Jiqiren/Robot, 2010: p534-539.

[8] Chen, Qin G. Robot inverse kinematics algorithm based on multi-agent system [J]. Electronics and Information Engineering, 2013 (v 347-350): p501-504.

[9] Jon Gress. Visual effects and compositing [M]. New Jersey Pearson education publishing group, 2014: p36-60.

[10] Ron Brinkmann, Visual Effects, Animation \& Motion Graphics [M] Tsinghua university press, 2012: p88-98. 